\title{
Explaining Loss Caused by Tomato spotted wilt virus on Tobacco with Boreal Winter Weather: A Bayesian Approach
}

\author{
A. L. Mila \\ Department of Plant Pathology, North Carolina State University, Campus Box 7405, Raleigh 27695. \\ Accepted for publication 11 November 2010.
}

\begin{abstract}
Mila, A. L. 2011. Explaining loss caused by Tomato spotted wilt virus on tobacco with boreal winter weather: A Bayesian approach. Phytopathology 101:462-469.

In North Carolina, Tomato spotted wilt virus (TSWV) has regularly been reported since 1997, with incidence being the highest in 2002. At the end of each season, a questionnaire is sent to the county agents to report disease losses. TSWV reported losses in 1993 to 2007 from 58 counties were available. A county-year combination was considered a case and, in total, 494 cases were analyzed. The winter months'

$0.0001)$ whereas the total precipitation for the same months had a negative effect $(P<0.0001)$. Bayesian hierarchical models were implemented to include spatial and nonspatial random effects to investigate if there were significant spatial correlations or unexplained variability, respectively, and, thus, other significant variables that were ignored in the model development. The spatial random effects were not significant but the nonspatial random effects were significant in 36 cases. The importance of spring weather to dispersal of thrips and TSWV has been previously identified. Winter weather also may be a good indicator of potential available TSWV inoculum for the upcoming season.
\end{abstract} temperature and precipitation significantly explained the reported TSWV loss $\left(R^{2}=0.82\right)$. Specifically, the monthly average air temperature for December to February had a positive association with TSWV loss $(P<$
Additional keywords: posterior distribution, random error.
Tomato spotted wilt virus (TSWV) was first reported in North Carolina in 1988 in tobacco (Nicotiana tabacum L.) and tomato (Lycopersicon esculentum Mill.) fields (6). Since 1997, damage by TSWV on tobacco has regularly been reported at economically damaging levels in several counties (T. Melton, personal communication). In 2002, TSWV incidence reached the highest historical levels in tobacco, with incidence as high as $50 \%$ in several fields (17).

TSWV is vectored by thrips. In North Carolina, Frankliniella fusca (Hunds) is the primary vector $(6,9)$. Commonly, spread of TSWV occurs due to infections by viruliferous adult thrips immigrating to a crop from outside sources, such as weeds located around tobacco fields $(6,11)$. Weather conditions prior to and during the growing season are critical for thrips development and movement and spread of TSWV. Spring precipitation is an important factor $(2,19)$. In Georgia, heavy rain during March and April resulted in low numbers of infections until mid-May (2). In North Carolina, total precipitation and days that rain occurred during January to May were significant variables explaining the variation in dispersing patterns of thrips (19).

Temperature is another major factor that determines development of thrips $(4,6)$. In a study conducted in North Carolina (6), population of thrips was lower in the western than in the eastern part of the state. Winter and spring temperatures were also lower in the west than in the east, implying a correlation between temperature and thrips dynamics (6). The lower temperature threshold for thrips populations growth is $10.5^{\circ} \mathrm{C}$ (16). In southeastern North Carolina, where TSWV loss on tobacco is the highest,

Corresponding author: A. L. Mila; E-mail address: almila@ncsu.edu

* The $\boldsymbol{e}$-Xtra logo stands for "electronic extra" and indicates that the online version contains one supplemental table.

doi:10.1094/PHYTO-05-10-0146

(C) 2011 The American Phytopathological Society temperature is rarely below this threshold. Thus, there may be a year-round reproduction of thrips on hosts other than tobacco in this part of the state (6). In Georgia, widespread TSWV incidence on tobacco was first observed in 1989 (8). The authors attributed the epidemic to the mild winter temperatures during 1988-89 that may have allowed survival of greater than normal numbers of weed hosts of TSWV that serve also as a host for thrips (8). Thus, higher levels of TSWV inoculum might have been available in 1989 than inoculum available in a season following a cold winter (8).

The application of pesticides has been the only successful tactic to manage TSWV on tobacco (7). Specifically recommended pesticides include imidacloprid (Admire Pro; Bayer Crop Science, Research Triangle Park, NC) or acibenzolar-S-methyl (Actigard 50 WG; Syngenta Crop Protection Inc., Greensboro, NC). Application of imidacloprid is associated with costs that growers are reluctant to assume, and only in cases when it is necessary. Furthermore, reduced plant growth, or even plant mortality, has been reported in the field with acibenzolar-S-methyl application (7). This limits acibenzolar-S-methyl application only to cases that TSWV incidence is so high that the control provided by acibenzolar-S-methyl offsets the potential for an adverse effect on tobacco growth.

Both imidacloprid and acibenzolar-S-methyl are applied in the greenhouse a few days before tobacco is transplanted to the field where TSWV infections occur (17). Thus, it is important to know the potential for TSWV occurrence several weeks before it actually happens. For instance, in 2002, a severe TSWV year, the disease incidence in several fields in North Carolina was $>20 \%$, even in counties of the northeastern part of the state (T. Melton, personal communication). These counties do not report high TSWV losses in most years, including years after the 2002 epidemic. Thus, growers recognize there is a potential for high TSWV incidence in their area; however, they commonly prefer to assume the risk of an outbreak of TSWV rather than use acibenzolar-S-methyl. Nevertheless, under high TSWV incidence, 
yield losses may occur and, thus, use of acibenzolar-S-methyl would be warranted.

A model that would predict TSWV occurrence some time in late winter or early spring, before transplanting tobacco to a field, would be very useful because it would provide ample time for decisions on TSWV management. Although it is of primary importance to know the expected occurrence in a given field, TSWV incidence is very variable even between fields located a few kilometers apart $(2,20)$. Therefore, its prediction may require collection of site-specific weather and other information that could demand substantial time from growers. A prediction for a large spatial unit, such as a county, could provide information useful for disease control decisions without a large effort from growers.

Whenever a model is developed, it is important that the underlying assumptions are scrutinized (24). For instance, for a model predicting TSWV occurrence at the county level, it is of interest to identify counties corresponding to extreme high or low unexplained occurrence to determine whether observed extrema are too large or small to have occurred by chance in the developed model. The model could be incorrect either because an important variable has been omitted or because one of the statistical assumptions is inappropriate (24). The investigation of underlying assumptions is often called model diagnostics and this is the term that will be used in the rest of this study (24).

The objective of the present study was to investigate whether boreal winter weather variables, specifically air temperature and total precipitation, significantly explained TSWV loss on tobacco. For this investigation, a correlation analysis between air temperature and total precipitation for each month between September (before planting) and August (during the season of interest) and TSWV loss reported in tobacco on a county level was conducted. The findings from the correlation analysis were used to develop an explanatory model for TSWV loss reported at the county level, and Bayesian diagnostic methods, commonly applied in human epidemiology, were used to examine whether assumptions made were appropriate or important variables were ignored in model development.

\section{MATERIALS AND METHODS}

Data collection. At the end of each growing season, a questionnaire is sent to county extension agents with tobacco responsibilities requesting detailed information on cultivars planted, crop rotation, fertilization, weed management, disease loss, chemical disease control, insect management, irrigation, sucker control, and fuel usage on tobacco production in their county. Information is requested as a percentage of tobacco acreages in a county. Due to the quota system on tobacco, detailed crop records have been collected on an annual basis since 1964. In case of disease losses, tobacco extension agents collected records by surveying fields in their counties to estimate incidence of tobacco diseases to verify their general appraisal of the crop situation in their county to reduce possible variability of this subjective appraisal (C. Main, personal communication). Disease loss is defined as a combination of TSWV prevalence (percentage of production area where disease occurs) and intensity of the disease within tobacco fields (number of symptomatic plants and severity of symptoms). For the present study, records for 1993 to 2007 were used because, before 1993, no county in North Carolina had reported TSWV losses. Data from 58 counties, which represent $95 \%$ of the counties in North Carolina where tobacco is produced, were used. Records for 5 to 14 years were available from each of these counties. Missing reports were from years with no county agent in a particular county. For 29 of the 58 counties, the same county agent reported losses every year since 1993 . In total, $77 \%$ of the records are from before 2003, reflecting a reduction in number of county agents between 2004 and 2007 due to retirement and delays in replacement.
Model development. Total precipitation and average air temperature for each month between September (before planting) and August (during the season of interest) were selected as input variables because these are easily accessible weather variables. Weather data were obtained from the North Carolina Climate Office (NCCO) website. NCCO has established a weather station in each county.

Initially, the correlation of each month's total precipitation and average air temperature with the TSWV reported loss was examined (using the Pearson's correlation coefficient, CORR procedure in SAS; SAS Institute, Inc., Cary, NC). For this initial analysis, data of reported TSWV loss since 1993 from 20 counties of eastern North Carolina where TSWV losses are commonly reported were used. The remaining 38 counties reported zero or close to zero loss every year; therefore, this initial analysis was not conducted for data from these counties. Correlations were investigated for each of the 20 counties separately for the total number of available records per county ( $n=7$ to 15 ).

As a second step, TSWV reported loss $y$ was logit transformed $(\ln [y /(100-y)])$ and modeled as a function of the winter weather variables (12). The logistic regression analysis was performed for each of the 20 counties of the eastern North Carolina separately. The LOGISTIC procedure (SAS Institute, Inc.) was used. To select the best set of predictors, the backward selection was used. This is an automatic procedure where, starting from a saturated model, no significant parameters are dropped, so that parameters in the final model are significantly associated with TSWV loss. The $c$ value, an analogue of the coefficient of determination $R^{2}$ of the ordinary least square regression, was used to evaluate goodness of fit. The $c$ value is equal to the area under a receiver operating characteristic curve and provides a measure of the predictive accuracy of a model and takes values between 0 and 1 (12). A model with higher values for this index has better predictive ability than a model with lower values for this index (12).

Last, TSWV loss data reported from all 58 counties and for all available years of records were analyzed together. A record with a unique combination of year and county was defined as a case. In total, 495 cases reported in 1993 to 2007 were used for model development. The sum of air temperature of December to February and the total precipitation of December, January, and February, used as inputs in the second step for each of the 20 counties of eastern North Carolina, were used and TSWV loss $y$ was logit transformed and modeled as

$$
\ln (y /(100-y))=\beta_{o}+\beta X
$$

where $\beta_{o}$ is the intercept and $\beta$ a vector of parameters to be estimated by maximum likelihood methods (12). The LOGISTIC procedure (SAS Institute, Inc.) was used. Goodness of fit was evaluated using the c value (12).

Bayesian model diagnostics. In each county $i, y_{i}$ is the reported TSWV loss and $e_{i}$ is the expected TSWV loss, predicted with the model described in equation 1 . The maximum likelihood estimate of the ratio $\left(r_{i}\right)$ is called the standardized mortality ratio (SMR) in human epidemiology for the $i$ th county $\left(r_{i}=y_{i} / e_{i}\right)$, with estimated standard error $s_{i}=\sqrt{ } y_{i} / e_{i}(15)$.

SMRs are commonly used in disease map presentation to summarize observed (or reported) versus expected risk (or loss) but have many drawbacks. First, SMRs are based on ratios and, hence, can yield large changes in estimate with relatively small changes in expected value $\left(e_{i}\right)(15)$. When a close to zero expectation is found, the SMR will be very large for any observed (or reported) value. Also, zero SMRs due to a zero observed or reported value, as in several counties and years in this study, do not distinguish variation in expected risk (or loss) (15).

In a simple modeling approach, such as the one described above, it is assumed that, once all known confounding variables 
are included and the appropriate assumptions for model development are made, then the resulting SMRs will be clean of artifacts and, thus, depict the true excess loss (15). Within a county, however, this may not be true because of extra variation components which are estimable but have not been included in the model. These components may have different forms depending on the degree of prior knowledge concerning the nature of this variation (15). There are two basic forms of extra variation or random effects. First, a form of independent and, thus, spatially uncorrelated variation can be assumed. Another form of variation arises when it is thought that a spatial unit is correlated with neighboring spatial units (15). This spatial correlation between counties could arise for a variety of reasons. First, TSWV loss in tobacco is higher in the southeastern counties of North Carolina than the rest of the state and, thus, counties in the southeast could be spatially more correlated than counties in another part of the state. Second, correlation can be induced in spatial patterns by the existence of unobserved environmental or even social effects that were not captured by the variables that were included in the analysis. An example in our study could be exchange of information and other observations between county agents working in neighboring counties that could influence their assessment of TSWV loss.

There are several advantages in modeling random effects within a Bayesian framework; therefore, its implementation has dominated the relevant statistical literature since the mid-1990s $(1,18)$. First, parameters are treated as random variables and, thus, parameter density is allowed to have any shape, possibly skewed or even multimodal. Second, modeling follows a hierarchical structure and assumptions can be scrutinized in each level of hierarchy separately as if this was an independent model. For instance, if spatial correlations are examined, then assumptions can be made about the degree of these correlations, such as first degree (i.e., if a point is correlated with a neighboring point) or second degree (i.e., if a point is correlated with two points apart from it). Similarly, if no spatial random effects are assumed and there is evidence that not all points of interest share the same probability $p$ for these random effects, then we can model separate probabilities $\left(p_{i}\right)$ for different effects. To answer some of these questions is particularly important when data are derived from subjective appraisals such as in the present study, where variability and uncertainly of the actual observation may vary among counties (18).

Here, I implemented a three-stage hierarchical Bayesian model proposed by Besag et al. (1). Reported loss $y_{i}$ was assumed to follow a binomial distribution, conditional on the underlying SMR $r_{i}$ for the county $i$. Often, the binomial is approximated by the Poisson distribution:

$$
y_{i} \approx \operatorname{Poisson}\left(e_{i} r_{i}\right)
$$

when $r_{i}$ is small and the population at risk is large; $e_{i} r_{i}$ is the expected TSWV loss and can be estimated using equation 1. Then,

$$
\log \left(y_{i}\right)=\log \left(e_{i} r_{i}\right)+V+U
$$

$V$ represents an extra variation with no spatial structure (so that $V_{i}$ and $V_{j}$ were independent for $i \neq j$ ), and $U$ represents an extra variation with spatial structure (so that $U_{i}$ and $U_{j}$ corresponded to neighboring counties and were modeled assuming to have positive spatial dependence) (24). In the second stage of modeling, it was assumed that $V \approx$ i.i.d. $N\left(0, \sigma_{v}{ }^{2}\right)$, where i.i.d. stands for independent and identically distributed. For $U$, often the conditional autoregressive Markov random field model has been used (1). This model is given by $U_{i} \mid U_{j} i \neq j \approx N\left(\bar{u}, \omega_{\mu}{ }^{2} / m_{i}\right)$ where $\bar{u}$ denotes the average value of the "neighbors" of county $i$, and $m_{i}$ is the number of such neighbors (1). In the present study, counties that share a common boundary were defined as neighbors (i.e., first degree correlation).

The third stage involved definition of the hyperparameters, that is, the parameter distributions of the prior distributions. First, we assumed no prior knowledge and, thus, noninformative distributions were assumed. Second, informative and very informative distributions were imposed. Commonly, the $\gamma$ distribution is used for the precision parameter $\tau=\sigma^{-2}$, where $\sigma^{-2}$ is the inverse of the variance terms. The $\gamma$ distribution is advantageous because it enables the convergence of the algorithm and, thus, the derivation of posterior distributions (1). Priors such as $\tau \approx \gamma(0.00001$, $0.0001)$ are noninformative, whereas $\tau \approx \gamma(0.1,0.1)$ or $\tau \approx \gamma(0.5$, $0.0005)$ express strong or very strong specifications, respectively. Imposing informative or very informative specifications and comparing the posterior distributions to the ones generated with noninformative specifications was used as means of sensitivity analysis. If the posterior parameter distributions were not significantly different when noninformative or informative prior distributions were used, then parameter estimates were not sensitive to the choice of priors. The inference is that reported data are sufficient to be used for robust inference (i.e., posterior parameter distributions and their credible intervals).

Bayesian model fitting and diagnostics were performed with Bayesian Using Gibbs Sampling software (WinBugs, version 1.4). Algorithm convergence is an important step in Bayesian analysis because the last $N$ iterations after convergence are used to calculate components describing parameter distributions (such as mean, median, standard deviation, and percentiles). Visual examination of trace plots, autocorrelations, and Gelman-Rubin diagnostic were applied to verify convergence. I performed two parallel runs of Gibbs sampler of 15,000 iterations, and the final 5,000 iterations were used to calculate the median and the 2.5 to 97.5th percentiles of the posterior distribution of the parameters.

Significance of posterior distributions was assessed by examining whether zero is included in the $95 \%$ credible intervals of the posterior distribution. Models using noninformative, informative, or very informative priors were compared with the use of deviance information criterion (DIC) (23). DIC is a generalization of Akaike's information criterion (AIC). The model with the smallest DIC is the model that would best predict a replicate dataset that has the same structure as that currently observed (23). Roughly, differences in DIC of $>10$ usually rule out the models with the higher DIC; differences in DIC of 5 to 10 are substantial and generally rule out the models with higher DIC. If the difference in DIC is $<5$, then it could be misleading just to report the model with the lowest DIC, especially if the models with similar DIC values produce different predictors or lead to different inferences (23).

\section{RESULTS}

Significance of boreal winter weather variables and model development. In 12 of the 20 counties of eastern North Carolina, TSWV losses were $>10 \%$ in several tobacco fields in 2002, the most severe TSWV epidemic year, but only isolated cases of high incidence were reported otherwise (Table 1, "Only in 2002" county group). There were eight counties that regularly report high TSWV losses (i.e., several fields with losses $>20 \%$ ) since 1993 (Table 1, "Every year" county group).

For each of the 20 counties, there was a high correlation of TSWV reported loss with the average air temperature and total precipitation of the boreal winter months (December, January, and February) (Table 2). Correlations ranged from $r=-0.24(P=$ $0.47)$ to $r=-0.88(P=0.01)$ for December precipitation, $r=$ $-0.14(P=0.61)$ to $r=-0.76(P=0.04)$ for January precipitation, and $r=-0.19(P=0.52)$ to $r=-0.56(P=0.06)$ for February precipitation. For monthly air temperature, these correlations were $r=0.22(P=0.44)$ to $r=-0.80(P=0.03)$ for December, $r=$ 
$-0.65(P=0.03)$ to $r=0.11(P=0.74)$ for January, and $r=-0.12$ $(P=0.69)$ to $r=0.59(P=0.05)$ for February. For the majority of the counties, the signs of these correlations were also consistent; that is, positive for average air temperature and negative for total precipitation (Supplementary Table 1).

The boreal winter weather variables were used as inputs to develop models explaining the TSWV reported loss in each of the 20 counties investigated. The results of the logistic regression analysis revealed that the air temperature and total precipitation for the months of December to February explained a significant portion of the variation in TSWV loss reported in each of the 20 counties. In Table 1 , only significant variables at $P=0.05$ were included. These results also revealed a consistent pattern. For the counties that regularly experience high TSWV losses (Table 1, "Every year" county group), both air temperature and total precipitation were important explanatory variables. Also, the signs of parameter estimates were consistent; that is, signs were negative for estimates related to precipitation and positive for estimates related to temperature. The exception was the sign for the total precipitation parameter for Pender and Sampson Counties (Table 1). For counties that reported significant TSWV loss in 2002 and sporadic outbreaks otherwise (Table 1, "Only in 2002" county group), the sign was negative for estimates related to precipitation (with the exception of Cumberland and Nash Counties) and positive for estimates related to temperature (with the exception of Nash County). Air temperature was an important explanatory variable in six counties whereas total precipitation was always significant (Table 1). Models had good explanatory power ranging from $c=0.66$ in Pender County to $c=0.90$ in Montgomery County (Table 1).

The results of the final analysis, where data from all counties and years were analyzed together, are presented in Table 2 and follow the general pattern of the results obtained for individual counties when investigated separately. That is, the sum of monthly average air temperature for the 3 months of winter had a positive effect on TSWV losses whereas the total precipitation for the same months had a significant negative effect. The $c$ value $=$ 0.82 . The $R^{2}$ values between reported TSWV loss and loss predicted by the model was 0.41 (Fig. 1A). The intercept of the regression between predicted and reported values was signifi- cantly different than zero $(P=0.001)$ and the slope was significantly different than $1(P=0.042)$. A difference $>3 \%$ between reported and predicted TSWV loss was found in 38 cases. These cases were $7.2 \%$ of the total cases used in the analysis; specifically, in 1993 for Jones County; in 1997 for Bertie, Columbus, Chowan, Duplin, Brunswick, Pender, Washington, and Wayne Counties; in 1999 for Lenoir County; in 2000 for Pender, Beaufort, and Onslow Counties; in 2001 for Columbus, Craven, Martin, and Pender Counties; in 2002 for Columbus, Craven, Greene, Lenoir, Martin, Pender, and Robeson Counties; in 2003 for Nash County; in 2004 for Jones, Pender, and Sampson Counties; in 2005 for Columbus, Craven, Lenoir, and Sampson Counties; in 2006 for Craven, Lenoir, Duplin, and Pender Counties; and in 2007 for Craven and Duplin Counties. The largest differences between reported and predicted TSWV loss was in cases since 2002. Interestingly enough, in 2002, cases where the difference between reported and predicted loss was substantially large, the reported loss was higher than the predicted, an indication that, during the severe TSWV epidemic year, the high and widespread incidence of the disease may have biased tobacco agents' assessment, or that the model tends to underestimate a severe epidemic.

Bayesian analysis and model diagnostics. The SMRs had a mean of 1.19 and ranged widely, from 0.0 for several counties in the west and Piedmont of North Carolina, where TSWV loss has rarely been reported, to 52.2 in Nash County in 2003 (Fig. 2). The standard errors of the SMRs ranged from 0.0 for counties with

TABLE 2. Parameter estimates of the logistic regression model used to explain Tomato spotted wilt virus loss reported on tobacco in 58 counties in North Carolina in 1993 to 2007

\begin{tabular}{lcc}
\hline Parameter & Estimate $^{\mathrm{a}}$ & $\mathrm{SE}^{\mathrm{b}}$ \\
\hline Intercept & -20.4 & 0.67 \\
Total precipitation of December & -0.007 & 0.001 \\
Total precipitation of January & -0.014 & 0.001 \\
Total precipitation of February & -0.008 & 0.001 \\
Sum of monthly average temperature $^{\mathrm{c}}$ & 0.14 & 0.005 \\
\hline
\end{tabular}

a All estimates are significant at $P=0.0001$.

${ }^{\mathrm{b}}$ Standard error.

${ }^{c}$ For December, January, and February.

TABLE 1. Parameter estimates of logistic regression models used to explain Tomato spotted wilt virus (TSWV) reported loss on tobacco with boreal winter weather variables in 20 counties of North Carolina in 1993 to 2007

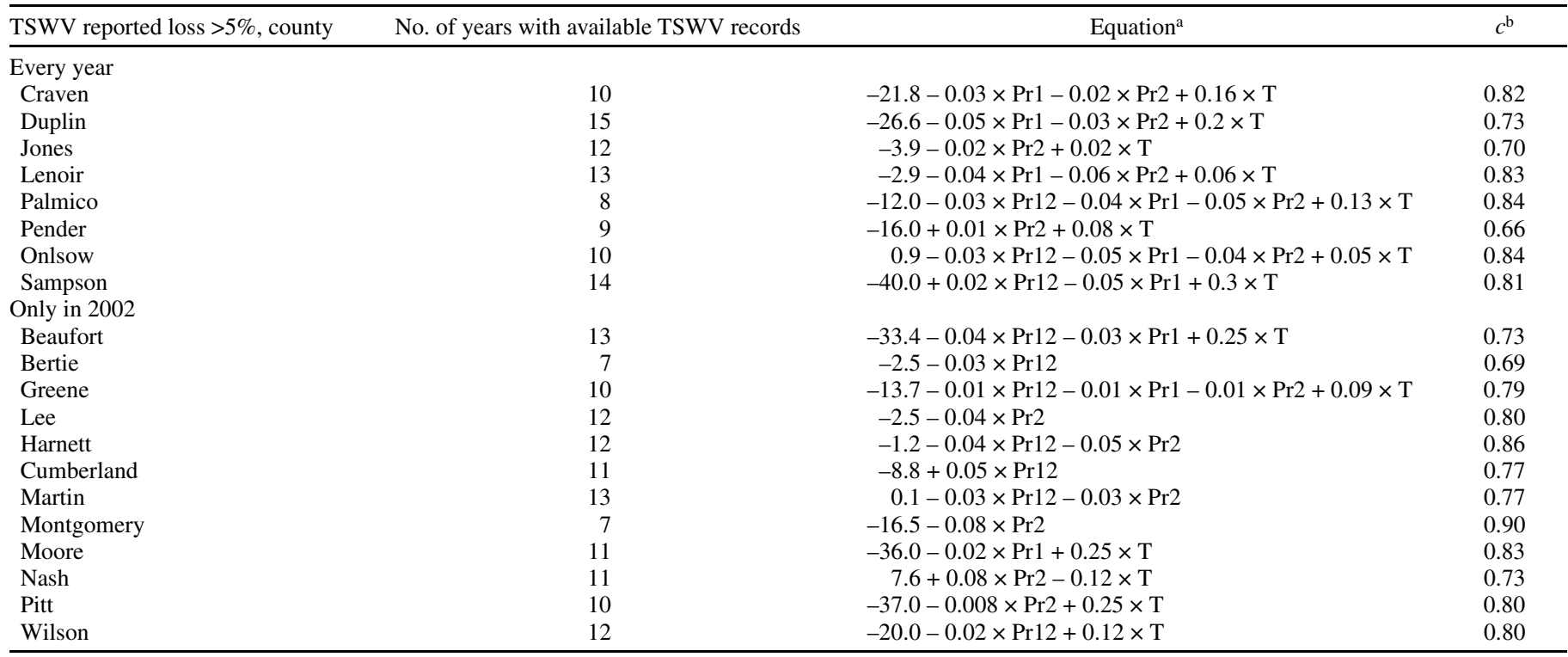

${ }^{\text {a }}$ Parameter estimates significantly different than zero $(P=0.05)$. Input variables were: $\mathrm{T}=$ sum of monthly average air temperature during December to February, $\operatorname{Pr} 12=$ total precipitation of December, $\operatorname{Pr} 1=$ total precipitation of January, and Pr2 = total precipitation of February.

${ }^{\mathrm{b}}$ Equal to the area under a receiver operating characteristic curve that measures the predictive accuracy of a model and takes values between 0 and 1 . A model with higher $c$ value has better predictive ability than a model with a lower value. 
very low predicted TSWV loss to 24.5 for Nash County in 2003. Examples of SMRs are shown in Figure 2; there is some suggestion of lower loss in western North Carolina and higher in the south and east, yet no other clear spatial pattern was apparent from the maps of SMRs.

Variation due to spatial effect $U$ was not significant in any case. The $95 \%$ of the credible interval for posterior distributions for spatial effect included 0 in all cases. Also, the use of informative priors did not significantly influence the posterior distribution of spatial effect; the difference in DIC was $<5$. On the other hand, nonspatial variation $V_{i}$ was significant in 36 cases (Table 3 ). In these cases, $95 \%$ of the credible interval of posterior distributions of nonspatial variation did not include 0 . These cases were $7 \%$ of the total cases used for the analysis. Of these cases, 26 were the same as those where there was $>3 \%$ difference between observed and predicted TSWV loss (Fig. 1A). There was no significant difference between the $95 \%$ credible interval of posterior distributions of TSWV loss when noninformative or very informative prior distributions were imposed on $V_{i}$, suggesting that the available data provided adequate information for robust inference. The $R^{2}$ values between observed and predicted TSWV loss when nonspatial variation was included in the model was 0.83 (Fig. 1B). The intercept of the regression between predicted and reported values was significantly different than zero $(P=0.001)$ and the slope was significantly different than $1(P=0.001)$.
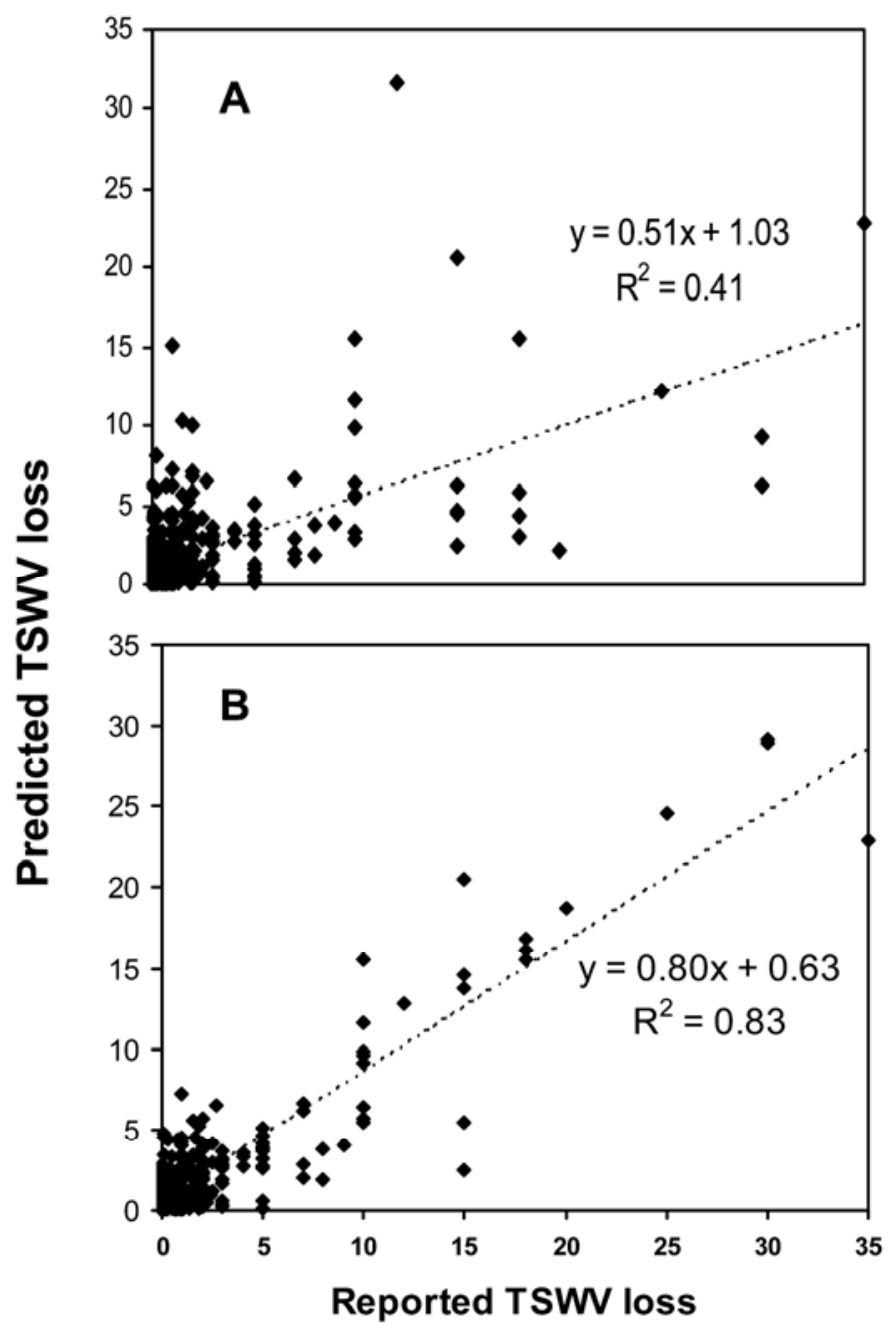

Fig. 1. Comparison of the predicted and the reported Tomato spotted wilt virus (TSWV) loss on tobacco for 58 counties in 1993 to 2007 in North Carolina with A, total precipitation and air temperature of December to February as explanatory variables and $\mathbf{B}$, the same variables and inclusion of nonspatial random effects. Model parameter estimates are presented in Table 2.
An example of medians and the 2.5 to 97.5 th percentiles of the posterior distributions of spatial and nonspatial random effects is presented in Table 4 for six counties for 2002 and 2003. These 2 years and counties were chosen because they represent a year of high (2002) and a year of low (2003) TSWV reported loss. If the range of the 2.5 to 97.5 th percentile of the posterior distribution does not include 0 , then the coefficient is significant. Posterior distributions of spatial variation coefficients were not significant for any county in both years (Table 4). In 2002, nonspatial variation was significant for Craven, Marin, and Palmico counties whereas, in 2003, no county had a significant nonspatial variation. The posterior distribution of the predicted TSWV loss in Duplin and Sampson Counties in 2005 is demonstrated in Figure 3. These
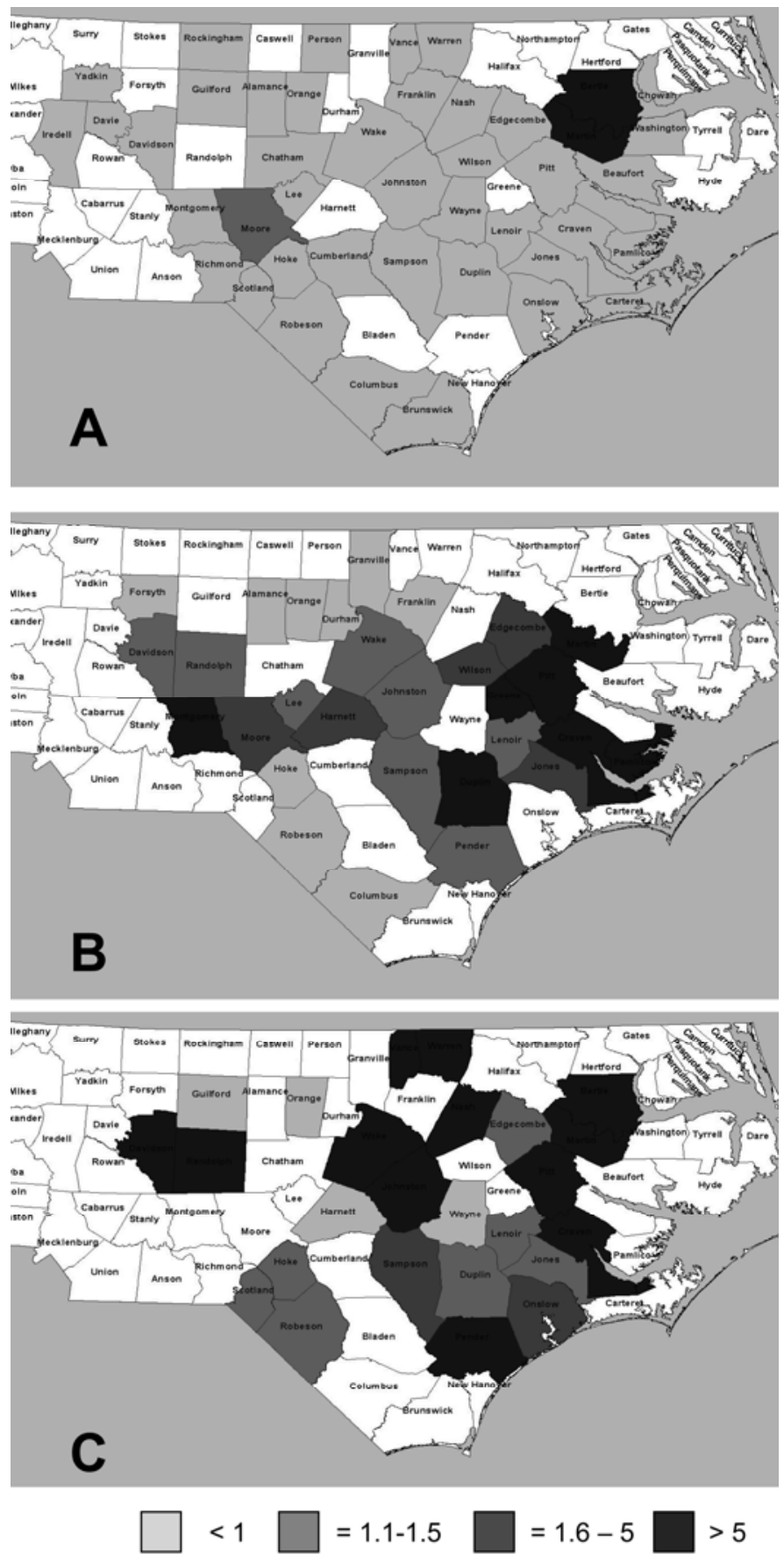

Fig. 2. Examples of standardized mortality ratios (SMRs) for counties in North Carolina during A, 1993; B, 2002; and C, 2003. SMRs are the ratio of reported to predicted Tomato spotted wilt virus loss, where a predicted loss was calculated with the model developed using boreal winter weather variables. Model parameter estimates are presented in Table 2. 
two counties share a common border. Also, they both reported $10 \%$ TSWV loss in 2005. Nonspatial variation was significant for Sampson but not for Duplin County. When nonspatial variation was included in the model, the $95 \%$ credible interval of the posterior distribution of predicted TSWV loss for Duplin County was 4.8 to $16.3 \%$, with a median of $9.2 \%$. Similarly, the $95 \%$ credible interval of the posterior distribution of predicted TSWV loss for Sampson County was 4.5 to $15.7 \%$, with a median of $8.8 \%$. Although the medians and the credible intervals were similar, a visual inspection of the posterior distributions of predicted TSWV losses revealed a different shape for the two counties: a normal shape for Duplin County and a right skewed one for Sampson County, a result of the presence of significant nonspatial variation in Sampson County.

\section{DISCUSSION}

The current study, based on reported TSWV loss from $95 \%$ of the counties where tobacco is grown in North Carolina in 1993 to 2007, revealed the importance of winter weather on the epidemiology of TSWV on tobacco. Dry and warm weather conditions during December to February were associated with more severe epidemics of TSWV compared with wet and cool conditions during the same months. The inclusion of spatial random effects with Bayesian methods revealed that there was no significant unexplained spatial variation. Nonspatial random effects were significant in 36 cases, all of which were in eastern North Carolina. In these cases, there was significant variation in re- ported TSWV loss that was not explained by the boreal winter months' weather.

Several studies have linked early spring weather conditions to changes in dynamics of vector populations that are particularly important for successful transmission of plant viruses $(14,19$, 21,22 ). Heavy rainfall was reported to have a negative effect on thrips larvae survival (14) and adult flight (19), whereas increased temperatures during the spring were associated with great thrips activity and population growth $(14,22)$. In peanut, an average daily minimum temperature $<6.8^{\circ} \mathrm{C}$ during March lowered the intensity of spotted wilt (21). Thrips may become inactive or stop reproducing at this temperature, which is much lower than the optimum temperature required for $F$. fusca $\left(24.9^{\circ} \mathrm{C}\right)$ oviposition (4). Olatinwo et al. (20) reported a significant correlation between degree-day accumulation (using $13.3^{\circ} \mathrm{C}$ as base and $28.9^{\circ} \mathrm{C}$ as maximum) and the severity of TWSV on peanut in Georgia. In the current study, degree-day accumulation was examined initially instead of average air temperature but did not improve model fit compared with air temperature. Therefore air temperature, a simple weather variable, was used for the analysis.

Studies have shown that F. fusca is common on winter annual plants during winter and early spring in the southeastern United States $(5,10,13)$. Groves et al. (10) found that both Stellaria media and Scleranthus annuus retained high levels of TSWV infection throughout the winter and spring in North Carolina. Typically, these winter-annual plants emerge over a prolonged period, beginning in early fall. Consequentially, weeds mature at different times during the spring. Hence, neighboring plants of the same

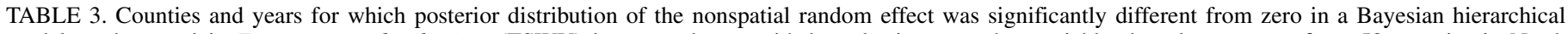

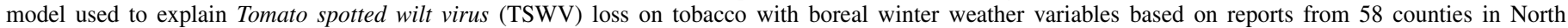
Carolina in 1993 to 2007

\begin{tabular}{|c|c|c|c|c|c|c|c|c|c|c|}
\hline \multirow[b]{2}{*}{ County } & \multicolumn{10}{|c|}{ Year $^{\mathrm{a}}$} \\
\hline & 1996 & 1997 & 1999 & 2000 & 2001 & 2002 & 2004 & 2005 & 2006 & 2007 \\
\hline Bertie & $\ldots$ & -* & $\ldots$ & $\ldots$ & $\ldots$ & $\ldots$ & $\ldots$ & $\ldots$ & $\ldots$ & $\ldots$ \\
\hline Chowan & $\ldots$ & -* & $\ldots$ & $\ldots$ & $\ldots$ & $\ldots$ & $\ldots$ & $\ldots$ & $\ldots$ & $\ldots$ \\
\hline Columbus & $\ldots$ & -* & $\ldots$ & $\ldots$ & -* & -* & $\ldots$ & -* & $\ldots$ & $\ldots$ \\
\hline Craven & $\ldots$ & $\ldots$ & $\ldots$ & $\ldots$ & $t^{*}$ & $+*$ & $\ldots$ & $+^{*}$ & $t^{*}$ & $+^{*}$ \\
\hline Greene & $\ldots$ & $\ldots$ & $\ldots$ & $\ldots$ & $\ldots$ & $t^{*}$ & $\ldots$ & $\ldots$ & $\ldots$ & $\ldots$ \\
\hline Lenoir & $\ldots$ & $\ldots$ & -* & $\ldots$ & $\ldots$ & $\ldots$ & $\ldots$ & $+^{*}$ & $+^{*}$ & $\ldots$ \\
\hline Martin & $\ldots$ & - & $\ldots$ & $\ldots$ & $+^{*}$ & $+*$ & + & $\ldots$ & $\ldots$ & $\ldots$ \\
\hline Pender & $\ldots$ & $t^{*}$ & $\ldots$ & $-*$ & -* & -* & $t^{*}$ & $\ldots$ & $\ldots$ & $\ldots$ \\
\hline Robeson & $\ldots$ & $\ldots$ & - & $\ldots$ & - & - & $\ldots$ & $\ldots$ & $\ldots$ & $\ldots$ \\
\hline Sampson & + & $\ldots$ & $\ldots$ & $\ldots$ & - & $\ldots$ & $+^{*}$ & $+^{*}$ & + & $\ldots$ \\
\hline Washington & $\ldots$ & -* & $\ldots$ & $\ldots$ & $\ldots$ & $\ldots$ & $\ldots$ & $\ldots$ & $\ldots$ & $\ldots$ \\
\hline Wayne & $\ldots$ & -* & $\ldots$ & - & - & $\ldots$ & $\ldots$ & $\ldots$ & $\ldots$ & $\ldots$ \\
\hline
\end{tabular}

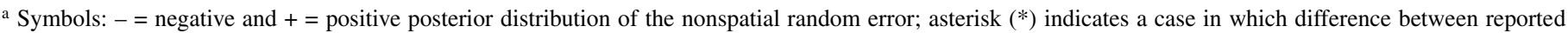
and predicted TSWV loss with the logistic regression model was $>3 \%$ and the posterior distribution of nonspatial random effect was significantly different from zero with a Bayesian hierarchical model.

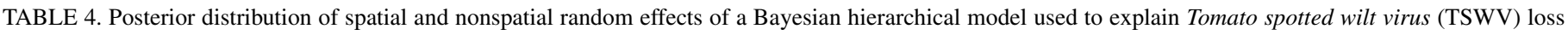
on tobacco in six counties of southeastern North Carolina in 2002 and $2003^{a}$

\begin{tabular}{|c|c|c|c|c|c|c|}
\hline \multirow[b]{2}{*}{ County, year ${ }^{b}$} & \multicolumn{3}{|c|}{ Spatial variation (U) } & \multicolumn{3}{|c|}{ Nonspatial variation (V) } \\
\hline & $2.5 \%$ & Median & $97.5 \%$ & $2.5 \%$ & Median & $97.5 \%$ \\
\hline Craven, 2002 & -0.55 & 0.80 & 2.05 & 0.27 & 0.69 & 1.06 \\
\hline Duplin, 2002 & -0.87 & 0.53 & 1.88 & -0.28 & 0.40 & 0.94 \\
\hline Lenoir, 2002 & -1.7 & -0.33 & 0.96 & -1.12 & -0.45 & 0.10 \\
\hline Martin, 2002 & -1.00 & 2.28 & 3.51 & 1.66 & 2.13 & 2.55 \\
\hline Palmico, 2002 & -2.17 & -0.77 & 0.51 & -1.56 & -0.96 & -0.47 \\
\hline Sampson, 2002 & -3.14 & -1.29 & 0.25 & -2.30 & -0.90 & 0.14 \\
\hline Craven, 2003 & -0.43 & 0.01 & 0.76 & -0.35 & 0.02 & 0.74 \\
\hline Duplin, 2003 & -0.37 & 0.01 & 0.72 & -0.56 & 0.00 & 0.58 \\
\hline Lenoir, 2003 & -0.47 & 0.00 & 0.58 & -0.71 & -0.01 & 0.38 \\
\hline Martin, 2003 & -0.58 & 0.00 & 0.46 & -0.45 & 0.01 & 0.81 \\
\hline Palmico, 2003 & -0.33 & 0.002 & 0.69 & -0.28 & 0.02 & 0.60 \\
\hline Sampson, 2003 & -0.59 & -0.01 & 0.41 & -0.48 & 0.00 & 0.64 \\
\hline
\end{tabular}

a If the range from the 2.5 to 97.5 th percentile of the posterior distribution does not include 0 , then the effect is significant.

${ }^{\mathrm{b}}$ In 2002, statewide reported TSWV loss was 6.3\% (a severe TSWV loss year) and, in 2003, TSWV loss was 0.7\% (a low TSWV loss year). 
species may pass through their most TSWV-susceptible stages on different dates. The incidence of TSWV infection would be greatest in those plants that were in the most susceptible stage during flights of viruliferous thrips and could result in localized patchiness in TSWV incidence (3).

In the present study, a relationship between winter weather and TSWV loss was established. A previous study has attributed high TSWV incidence on tobacco crops in Georgia to warm winter temperatures; however, quantification was not attempted (8). The positive effect of winter temperature on TSWV loss may be related to the abundant growth of winter weed hosts and the earlier initiation of trips reproduction $(5,10)$. Early initiation of reproduction may lead to higher population of thrips during spring but also larger population of weed hosts that are infected with TSWV (3). Similarly, the negative effect of precipitation during the same winter months may be related to a higher number of larvae and, thus, to a larger population of adult thrips during spring.

In previous research, the importance of spring weather to dispersal of thrips and virus has been identified (19); however, winter weather may be a good indicator of potential TSWV inoculum available for the following season. In Stewart's disease (Pantoea stewartii subsp. stewartii) of corn, for example, the Stevens Model attempts to predict the amount of initial inoculum available in early spring to initiate disease epidemics (25).
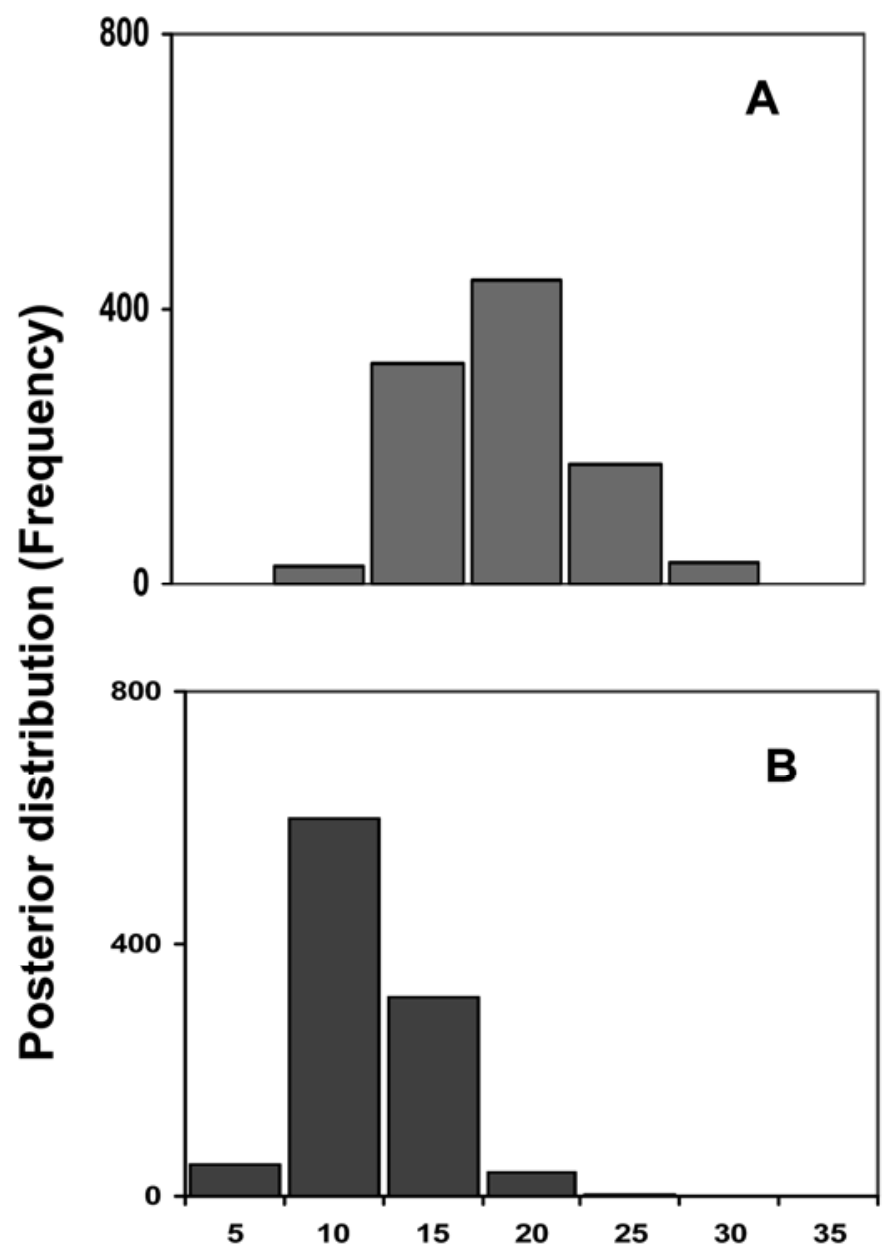

\section{TSWV percent loss}

Fig. 3. Posterior distribution of predicted Tomato spotted wilt virus (TSWV) loss in A, Duplin County and B, Sampson County in 2005. Counties were selected because both reported 10\% loss; however, spatial random effect was significantly different than zero for Sampson but not for Duplin County.
Disease risk is represented as number of $P$. stewartii-infested corn flea beetles that survived the winter. This risk is often a function of the effect of winter temperatures on survival of corn flea beetle populations. The Stevens Model (25) was developed by comparing differences in winter air temperatures for years in which Stewart's disease was severe with years when Stewart's was absent or of low severity. A similar relationship was revealed in the present study for another vector-transmitted disease, TSWV, of tobacco crops.

Given that data used to develop this model was from 14 years and over 50 counties that may be as far as $250 \mathrm{~km}$ apart, the prediction accuracy $\left(R^{2}=0.41\right)$ was reasonably good. I performed model diagnostics using Bayesian hierarchical methods that have become widespread in human disease mapping and ecological studies of health-environment associations $(1,15,18)$. In the present study, diagnostics were implemented to investigate whether the assumption of nonspatial correlations made during the development of the model of TSWV loss was appropriate and to determine whether reported extreme were too large or too small to have occurred by chance under the developed model.

Spatial effects were not significant for any year or county, indicating that the initial assumption of independence among county reports was valid and appropriate. Winter weather is different between areas of North Carolina; for instance, cooler in the western than in the eastern areas of the state. Weather variables were included in the model and, thus, spatial patterns may have been captured by these variables; therefore, no significant spatial extra variation was unexplained. Nonspatial random effect was significant in 36 cases and the prediction accuracy increased from 0.41 to 0.83 when nonspatial random effect was included in the model (Fig. 1B). These random effects could represent, among other things, high variability in disease assessment or the influence of field-scale environment and inoculum levels. For instance, in Sampson County, the distance between the first tobacco field in the south and the last one in the north is $55 \mathrm{~km}$ and TSWV loss ranged from $>20$ to $<5 \%$, respectively. Use of the 95 percentile range of posterior distribution for TSWV loss accounts for such uncertainty and provides a way to quantify range of loss instead of mean loss. This seems to be useful, particularly in the case of counties where TSWV loss range was wide, fluctuating around the mean county loss.

The model developed in this study predicts TSWV loss several weeks before the actual disease occurrence. Therefore, it provides substantial time for management decisions, particularly the necessity of imidacloprid or acibenzolar-S-methyl application in the greenhouse a few days before tobacco is transplanted to the field. It is based on observed weather and, therefore, it is not affected by uncertainty related to weather prediction that could reduce further prediction accuracy. As with all models, applicability and performance of this model should be evaluated before implementation. The model is under evaluation with data currently collected in North Carolina and other tobacco-producing states of the southeastern United States.

\section{ACKNOWLEDGMENTS}

I thank L. Madden for critically reviewing the manuscript before submission and the county agents of the North Carolina Cooperative Extension Services for reporting TSWV losses to the Tobacco Extension program of the Department of Plant Pathology in North Carolina State University.

\section{LITERATURE CITED}

1. Besag, J., York, J., and Mollie, A. 1991. Bayesian image restoration with two applications in spatial statistics. Ann. Inst. Stat. Math. 43:1-59.

2. Brown, S., Csinos, A., Diaz-Perez, J. C., Gitaitis, R., LaHue, S. S., Lewis, J., Martinez, N., McPherson, R., Mullis, S., Nischwitz, C., Riley, D., Sherwood, J., Sisson, V., Stephenson, M. G., and Wells, L. 2005. Tospo- 
viruses in solanaceae and other crops in the coastal plain of Georgia. Univ. Ga. Coll. Agric. Environ. Sci. Res. Rep. 704:I9.

3. Burdon, J. J., Jarosz, A. M., and Kirby, G. C. 1989. Pattern and patchiness in plant-pathogen interactions-causes and consequences. Annu. Rev. Ecol. Syst. 20:119-136.

4. Chaisuekul, C., and Riley, D. G. 2005. Host plant, temperature, and photoperiod effects on ovipositional preference of Frankliniella occidentalis and Frankliniella fusca (Thysanoptera:Thripidae). J. Econ. Entomol. 98:2107-2113.

5. Cho, J. J., Mau, R. F. L., Gonsalves, D., and Mitchell, W. C. 1986. Reservoir weed hosts of tomato spotted wilt virus. Plant Dis. 70:1014-1017.

6. Cho, K., Eckel, C. S., Walgenbach, J. F., and Kennedy, G. G. 1995. Overwintering of thrips (Thysanoptera: Thripidae) in North Carolina. Environ. Entomol. 24:58-67.

7. Csinos, A., Pappu, H., McPherson, R., and Stephenson, M. 2001. Management of Tomato spotted wilt virus in flue-cured tobacco with acibenzolar-S-methyl and imidacloprid. Plant Dis. 85:292-296.

8. Culbreath, A. K., Bertrand, P. F., and Demski, J. M. 1991. Tomato spotted wilt virus epidemic in flue-cured tobacco in Georgia. Plant Dis. 75:483485.

9. Eckel, C. S, Cho, K., Walgenbach, J. F., Kennedy, G. G., and Moyer, J. W. 1996. Variation in thrips species composition in field crops and implications for tomato spotted wilt epidemiology in North Carolina. Entomol. Exp. Appl. 78:19-29.

10. Groves, R. L., Walgenbach, J. F., Moyer, J. W., and Kennedy, G. G. 2001. Overwintering of Frankliniella fusca (Thysanoptera: Thripidae) on winter annual weeds infected with Tomato spotted wilt virus and patterns of virus movement between susceptible weed hosts. Phytopathology 91:891-899.

11. Groves, R. L., Walgenbach, J. F., Moyer, J. W., and Kennedy, G. G. 2002. The role of weed hosts and tobacco thrips, Frankliniella fusca, in the epidemiology of Tomato spotted wilt virus. Plant Dis. 86:573-582.

12. Hosmer, D. W., and Lemeshow, S. 2000. Applied Logistic Regression, 2nd ed. John Willey and Sons, New York.

13. Johnson, R. R., Black, L. L., Hobbs, H. A., and Valverde, R. A. 1995. Association of Frankliniella fusca and three winter weeds with tomato spotted wilt virus in Louisiana. Plant Dis. 79:572-576.
14. Kirk, W. D. J. 1997. Distribution, abundance, and population dynamics Pages 217-258 in: Thrips as Crop Pests. T. Lewis, ed. CAB International, New York.

15. Lawson, A. B. 2001. Disease map reconstruction. Stat. Med. 20:21832204.

16. Lowry, V. K., Smith, J. W., and Mitchell, F. L. 1992. Life-fertility tables for Frankliniella fusca (Hinds) and F. occidentalis (Pergrande) (Thysanoptera: Thripidae) on peanut. Ann. Entomol. Soc. Am. 85:744-754.

17. Mila, A. L., and Radcliff, J. 2009. Managing diseases. Pages 140-174 in: 2009 Flue-Cured Tobacco Production Guide. North Carolina State University, Cooperative Extension Services, College of Agriculture and Life Sciences, Raleigh, NC.

18. Mollie, A. 1996. Bayesian mapping of disease. Pages 359-379 in: Markov Chain Monte Carlo in Practice. W. R. Gilks, S. Richardson, and D. J. Spiegelhalter eds., Chapman and Hall, London.

19. Morsello, S. C., Groves, R. L., Nault, B. A., and Kennedy, G. G. 2008. Temperature and precipitation affect seasonal patterns of dispersing tobacco thrips, Frankliniella fusca, and onion thrips, Thrips tabaci (Thysanoptera: Thripidae) caught on sticky traps. Environ. Entomol. 37:79-86.

20. Olatinwo, R. O., Paz, J. O., Brown, S. L., Kemerait, R. C., Jr., Culbreath, A. K., Beasley, J. P., Jr., and Hoogenboom, G. 2008. A predictive model for spotted wilt epidemics in peanut based on local weather conditions and the Tomato spotted wilt virus risk. Phytopathology 98:1066-1074.

21. Olatinwo, R. O., Paz, J. O., Brown, S. L., Kemerait, R. C., Jr., Culbreath, A. K., and Hoogenboom, G. 2009. Impact of early spring weather factors on the risk of tomato spotted wilt in peanut. Plant Dis. 93:783-788.

22. Pearsall, I. A., and Myers, J. H. 2001. Spatial and temporal patters of dispersal of western flower thrips (Thysanoptera: Thripidae) in nectarine orchards in British Columbia. J. Econ. Entomol. 94:831-843.

23. Spiegelhalter, D. J., Best, N. G., Carlin, B. P., and Van der Linde, A. 2002. Bayesian measures of model complexity and fit (with Discussion). J. R. Stat. Soc. Ser. B 64:583-616.

24. Stern, H. S., and Cressie, N. A. 2000. Posterior predictive model checks for disease mapping models. Stat. Med. 19:2377-2397.

25. Stevens, N. E. 1934. Stewart's disease in relation to winter temperatures. Plant Dis. Rep. 18:141-149. 